LMU-TPW-97/14

hep-ph/9706278

\title{
Vector and Scalar Confinement in Gauge Theory with a Dilaton
}

\author{
Rainer Dick \\ Sektion Physik der Universität München \\ Theresienstr. 37, 80333 München, Germany
}

\begin{abstract}
In a recent letter it has been shown that gauge theory with a dilaton provides linearly increasing gauge potentials from static or uniformly moving pointlike colour sources. This ensures confinement in the framework of no-pair equations. Here I would like to point out that a dilaton coupling both to the gauge curvature term and to fermion masses yields a linear potential with a scalar component and a dominant vector contribution.
\end{abstract}


1. To understand confinement in gauge theories provides a challenge for theoretical physics since more than two decades. Many approaches to the problem rely on flux tube pictures, with flux tubes emerging between colour sources through gluon selfinteractions or through monopole condensation and the dual Meissner effect. Numerous investigations are dedicated to improve our understanding of this phenomenon and a collection of pioneering papers is [1]. Meson spectroscopy indicates a linear growth of energy with the distance between bound quarks and linear potentials are under close investigation as phenomenological models for quark confinement since many years, see [2] for early references. Lattice calculations have confirmed this picture by showing good agreement of a linear potential with a running coupling and nowadays are also used to probe the spin and momentum dependent parts of quark interactions [3]. Important approaches to an improved theoretical understanding of confinement in gauge theories have been further developed over the years, including semi-relativistic expansions of Wilson loops [4], dual QCD [5], and monopoles in $N=2$ supersymmetric gauge theories [6].

Recently it has also been pointed out that inclusion of a dilaton in gauge theories admits a solution of the Coulomb problem with a linearly increasing gauge potential [7]. An important classical difference between linearly increasing gauge potentials and $1 / r$ potentials concerns the fact that the quark self energy is not ultraviolet but infrared divergent, whence the divergence could not be attributed to new physics at short distances. This indicates that increasing vector potentials yield confinement. On the other hand, one would still like to confirm this in a picture of gluon-dilaton mediated quark-quark interactions, where we assume that at least one of the quarks is heavy enough to make potential confinement a reasonable assumption. It is well known that a linearly increasing 4 -vector potential is sufficient to ensure confinement in the framework of no-pair equations [8], and the assertion that the confining potential is a Lorentz vector received wide acceptance in recent years. On the other hand, it is known since long that a linearly increasing timelike component of a 4 -vector is not sufficient to ensure suppression of oscillatory solutions of the corresponding Dirac equation without projection operators. If one does not want to rely on no-pair equations the gauge potential has to be supplemented by a dominant scalar interaction to ensure confinement. I do not consider this as an attractive scenario, but I would like to point out that the dilaton can also accomodate a scalar contribution to the confinement potential if in addition to its coupling to $F^{2}$ it also couples to mass terms of quarks. The linearly increasing scalar potential then arises as a consequence of the logarithmic increase of the dilaton. However, we will see that the vector contribution dominates. Although dominance of a scalar contribution has been considered favourable for a while, dominance of the vector part saves us from a minor complication: Scalar dominance in the interquark potential should imply that all quark combinations are confined, with the phenomenologically required combinations only 
being energetically preferred. Nevertheless, by raising the quark masses above the coupling scale of the dilaton we can identify a simple model where quark confinement is realized even on the level of a naive Dirac equation.

Independently from the possibility to formulate models where confinement can be treated analytically, there exists strong motivation to include dilatonic degrees of freedom in gauge theory:

Maybe the oldest motivation comes from Kaluza-Klein theory, where the dilaton provides a measure for the size of internal dimensions. From a more modern point of view, the axion already provides two independent reasons to include a dilaton in gauge theories: If the gauge theory is supersymmetric the axion and the dilaton come together in a chiral multiplet, and even without supersymmetry the axion has to come with a dilaton if duality symmetry is realized. The massless spectrum of superstring theory provides yet another motivation to include a dilaton, and recent developments reviewed e.g. in [9] encompass all these reasons.

The possibility of a dilaton coupling to mass terms was not considered attractive until a few years ago, since generically such a coupling is expected to conflict constraints from the weak equivalence principle [10]. Indeed, for this reason I assumed that the dilaton does not couple to low energy mass terms, the justification for this assumption being that fundamental dilatons are expected to arise far above any low energy scale where low energy mass terms arise e.g. through spontaneous symmetry breaking. On the other hand, Damour and Polyakov have pointed out that even massless dilatons coupling to masses of other particles may be in agreement with current observational constraints for certain classes of coupling functions [11], and this motivated me to reconsider dilaton-mass couplings.

2. In the present paper I would like to focus on a model described by a Lagrange density

$$
\begin{gathered}
\mathcal{L}=-\frac{1}{4} \exp \left(\frac{\phi}{f_{\phi}}\right) F_{\mu \nu}{ }^{j} F^{\mu \nu}{ }_{j}-\frac{1}{2} \partial^{\mu} \phi \cdot \partial_{\mu} \phi \\
+\sum_{f=1}^{N_{f}} \bar{\psi}_{f}\left(i \gamma^{\mu} \partial_{\mu}+g \gamma^{\mu} A_{\mu}{ }^{j} X_{j}\right) \psi_{f}-\exp \left(-\xi \frac{\phi}{2 f_{\phi}}\right) \sum_{f=1}^{N_{f}} \bar{\psi}_{f} m_{f} \psi_{f},
\end{gathered}
$$

where $X_{j}$ denotes an $N_{c}$-dimensional representation of $\operatorname{su}\left(N_{c}\right)$. The particular form of the coupling functions of the dilaton to gauge fields and fermions is not completely artificial: For $\xi=1$ it corresponds exactly to what one would find in a Kaluza-Klein Ansatz connecting Einstein-Yang-Mills theory in $D>4$ dimensions to EinsteinYang-Mills-dilaton theory in four dimensions, where $\exp \left(2 \phi / f_{\phi}\right)$ is the determinant of the internal metric. 
The equations of motion are

$$
\begin{gathered}
\partial_{\mu}\left(\exp \left(\frac{\phi}{f_{\phi}}\right) F_{i}^{\mu \nu}\right)+g \exp \left(\frac{\phi}{f_{\phi}}\right) A_{\mu}{ }^{j} f_{i j}{ }^{k} F_{k}^{\mu \nu}=-g \bar{\psi} \gamma^{\nu} X_{i} \psi, \\
\partial^{2} \phi=\frac{1}{4 f_{\phi}} \exp \left(\frac{\phi}{f_{\phi}}\right) F_{\mu \nu}{ }^{j} F^{\mu \nu}{ }_{j}-\frac{\xi}{2 f_{\phi}} \exp \left(-\xi \frac{\phi}{2 f_{\phi}}\right) \bar{\psi} m \psi, \\
\left(i \gamma^{\mu} \partial_{\mu}+g \gamma^{\mu} A_{\mu}{ }^{j} X_{j}\right) \psi-\exp \left(-\xi \frac{\phi}{2 f_{\phi}}\right) m \psi=0
\end{gathered}
$$

Here and in the sequel flavour indices are suppressed.

We are interested in stationary colour distributions of the form

$$
j^{\mu}(x)=\varrho(\mathbf{r}) \eta_{0}^{\mu}=\rho(\mathbf{r}) C^{i} X_{i} \eta_{0}^{\mu}
$$

where $C_{i}$ is the expectation value of the generator $X_{i}$. Pointlike sources are of this type and the equations (2) reduce to

$$
\nabla \cdot\left(\exp \left(\frac{\phi}{f_{\phi}}\right) \nabla \Phi\right)=-\varrho
$$

with $\Phi \equiv A^{0}$. For $\rho(\mathbf{r})=g \delta(\mathbf{r})$ we have

$$
\exp \left(\frac{\phi(r)}{f_{\phi}}\right) \mathbf{E}_{i}(\mathbf{r})=\exp \left(\frac{\phi(r)}{f_{\phi}}\right) E_{i}(r) \mathbf{e}_{r}=\frac{g C_{i}}{4 \pi r^{2}} \mathbf{e}_{r}
$$

and we have to determine the dilaton from

$$
\frac{d^{2}}{d r^{2}} \phi(r)+\frac{2}{r} \frac{d}{d r} \phi(r)=-\frac{g^{2}}{64 \pi^{2} f_{\phi}}\left(1-\frac{1}{N_{c}}\right) \exp \left(-\frac{\phi(r)}{f_{\phi}}\right) \frac{1}{r^{4}}-\frac{\xi}{2 f_{\phi}} \exp \left(-\xi \frac{\phi}{2 f_{\phi}}\right) m \delta(\mathbf{r})
$$

where the property

$$
\sum_{i=1}^{N_{c}^{2}-1} C_{i}^{2}=\frac{N_{c}-1}{2 N_{c}}
$$

of expectation values in colour space was used. Using the abbreviation

$$
r_{\phi}=\frac{g}{8 \pi f_{\phi}} \sqrt{\frac{1}{2}-\frac{1}{2 N_{c}}}
$$

I would like to emphasize that both the regularized Coulomb potential discovered in case $\xi=0$ in $[12,7$

$$
\phi(r)=2 f_{\phi} \ln \left(\frac{r+r_{\phi}}{r}\right)
$$




$$
\Phi_{i}(r)=\frac{g C_{i}}{4 \pi\left(r+r_{\phi}\right)}
$$

and the confining solution [4]

$$
\begin{gathered}
\phi(r)=2 f_{\phi} \ln \left(\frac{r_{\phi}}{r}\right), \\
\Phi_{i}(r)=-\frac{g r}{4 \pi r_{\phi}^{2}} C_{i}
\end{gathered}
$$

persist for arbitrary values $\xi \geq 0$.

The resulting scalar potentials seen by the fermions in the two phases are

$$
S(r)=m\left(\frac{r}{r+r_{\phi}}\right)^{\xi}
$$

or

$$
S(r)=m\left(\frac{r}{r_{\phi}}\right)^{\xi}
$$

respectively.

3. To discuss the confining solution we assume that the potential is created by a heavy pointlike source with an orientation $\zeta_{s}$ in colour space, i.e.

$$
C_{i}=\zeta_{s}^{+} \cdot X_{i} \cdot \zeta_{s}
$$

Restricting $\xi$ to the values $\xi=0$ (no mass coupling) or $\xi=1$ (Kaluza-Klein type coupling) the net potential seen by an (anti-)quark of colour $\zeta_{q}$ in the rest frame of a heavy source is then

$$
V(r)=\left[ \pm \frac{g^{2}}{8 \pi r_{\phi}}\left(\left|\zeta_{s}^{+} \cdot \zeta_{q}\right|^{2}-\frac{1}{N_{c}}\right)+m \xi\right] \frac{r}{r_{\phi}} .
$$

The vector part implies that a blue source attracts an anti-blue quark in the sense that anti-quarks within an angle $\Theta_{c}=\arccos \left(\sqrt{1 / N_{c}}\right)$ from the positive or negative blue axis are attracted. Correspondingly a blue source attracts quarks of different colour in the sense that their colour orientation must lie outside the double cone defined by the angle $\Theta_{c}$.

The mass term, on the other hand, is always attractive, and we may ask under which circumstances we could expect strict confinement even in the framework of a naive Dirac equation: The tree level cross section for creation of a dilaton from head on collision of two gauge bosons with centre of mass energy $\sqrt{s}$ goes with $s / f_{\phi}^{4}$, and therefore we certainly expect

$$
m<g f_{\phi} \sqrt{2-\frac{2}{N_{c}}}
$$


no matter what definition of quark masses we would employ. Therefore, the vector part will always dominate and we should not expect scalar dominance in any realistic model. Nevertheless, I would also like to advertise the model with Kaluza-Klein type couplings and masses exceeding the coupling scale $f_{\phi}$ : These are models which provide confinement in the simplest possible setting through the Dirac equation, and we may learn something about thermodynamical aspects of confinement from these simple four-dimensional models.

Coming back to the vectorial part, we have seen that it is attractive for quarkantiquark pairs of the same colour and for quark pairs of different colour. Now suppose we have an ensemble of quarks forming a plasma with a mean separation $r$. Neglecting the scalar contribution, the energy gain $\epsilon_{b \bar{b}}$ in forming a blue-anti-blue meson exceeds the energy gain $\epsilon_{b r}$ in forming a red-blue diquark by a factor $N_{c}-1$, and therefore the gain in energy in forming $N_{c}$ mesons equals the gain in energy in forming a nucleon and an anti-nucleon. Hence, if the scalar contribution can be neglected and as long as quarks and anti-quarks appear with equal densities the plasma has no preference energetically to decay predominantly into one of the two channels. There nevertheless may appear asymmetries for three reasons: Kinematically the formation of two-particle bound states is favoured over the formation of $N_{c}$-particle bound states. On the other hand, the scalar contribution would favour nucleons, since the gain $2 E_{N}$ in forming a nucleon and an anti-nucleon with the mass couplings taken into account exceeds the gain $N_{c} E_{b \bar{b}}$ in forming $N_{c}$ mesons by $2 E_{N}=N_{c} E_{b \bar{b}}+N_{c}\left(N_{c}-2\right) \Delta$. Here $\Delta$ is the contribution from the scalar term to the binding energy of a meson or diquark. Finally, only nucleons would form after the anti-quarks have been used up.

\section{References}

[1] H.B. Nielsen and P. Olesen, Nucl. Phys. B61 (1973) 45;

H. Fritzsch and P. Minkowski, Phys. Lett. 49B (1974) 462;

J. Kogut and L. Susskind, Phys. Rev. D9 (1974) 3501;

G. Parisi, Phys. Rev. D11 (1975) 970;

G. 't Hooft, Nucl. Phys. B79 (1974) 276;

K.G. Wilson, Phys. Rev. D10 (1974) 2445.

[2] B.J. Harrington, S.Y. Park and A. Yildiz, Phys. Rev. Lett. 34 (1975) 168;

E. Eichten, K. Gottfried, T. Kinoshita, J. Kogut, K.D. Lane and T.-M. Yan, Phys. Rev. Lett. 34 (1975) 369;

H.J.W. Müller-Kirsten, Phys. Rev. D12 (1975) 1103;

J.F. Gunion and R.S. Willey, Phys. Rev. D12 (1975) 174;

K.S. Jhung, K.H. Chung and R.S. Willey, Phys. Rev. D12 (1975) 1999; 
J.S. Kang and H.J. Schnitzer, Phys. Rev. D12 (1975) 841, 2791;

J.F. Gunion and L.F. Li, Phys. Rev. D12 (1975) 3583, D13 (1976) 82;

T. Goldman and S. Yankielowicz, Phys. Rev. D12 (1975) 2910;

S.K. Bose, A. Jabs and H.J.W. Müller-Kirsten, Phys. Rev. D13 (1976) 1489.

[3] M. Creutz, Phys. Rev. D21 (1980) 2308;

M. Lüscher, K. Symanzik and P. Weisz, Nucl. Phys. B173 (1980) 365;

S.P. Booth, D.S. Henty, A. Hulsebos, A.C. Irving, C. Michael and P.W. Stephenson, Phys. Lett. B294 (1992) 385;

K.D. Born, E. Laermann, R. Sommer, T.F. Walsh and P.M. Zerwas, Phys. Lett. B329 (1994) 325;

G.S. Bali, K. Schilling and A. Wachter, Complete $\mathcal{O}\left(v^{2}\right)$ corrections to the static interquark potential from $S U$ (3) gauge theory, hep-lat/9703019.

[4] E. Eichten and F. Feinberg, Phys. Rev. D23 (1981) 2724;

N. Brambilla, P. Consoli and G.M. Prosperi, Phys. Rev. D50 (1994) 5878;

N. Brambilla and G.M. Prosperi, in Quark Confinement and the Hadron Spectrum, World Scientific, Singapore 1995, pp. 113-125.

[5] M. Baker, J.S. Ball and F. Zachariasen, Phys. Rep. 209 (1991) 73, Phys. Rev. D51 (1995) 1968.

[6] N. Seiberg and E. Witten, Nucl. Phys. B426 (1994) 19, B431 (1994) 484.

[7] R. Dick, Phys. Lett. B397 (1997) 193, Implications of a dilaton in gauge theory and cosmology, hep-th/9704193.

[8] J. Sucher, Phys. Rev. A22 (1980) 348, Phys. Rev. D51 (1995) 5965;

M.G. Olsson, S. Veseli and K. Williams, Phys. Rev. D51 (1995) 5079, D53 (1996) 4006.

[9] J.H. Schwarz, Lectures on superstring and M-theory dualities, hep-th/9607201; M. Dine, String theory dualities, hep-th/9609051;

C. Vafa, Lectures on strings and dualities, hep-th/9702201.

[10] A. De Rújula, Phys. Lett. B180 (1986) 213;

J. Ellis, S. Kalara, K.A. Olive and C. Wetterich, Phys. Lett. B228 (1989) 264.

[11] T. Damour and A.M. Polyakov, Nucl. Phys. B423 (1994) 532, Gen. Rel. Grav. 26 (1994) 1171.

[12] M. Cvetič and A.A. Tseytlin, Nucl. Phys. B416 (1994) 137. 